Revue d'histoire de l'Amérique française

6. REVUE D.HISTOIRE DE L'AMÉRIQUE FRANÇAISE

\title{
Des sujets de recherche, pour un siècle ou deux...
}

\section{Marcel Trudel}

Volume 20, numéro 2, septembre 1966

URI : https://id.erudit.org/iderudit/302567ar

DOI : https://doi.org/10.7202/302567ar

Aller au sommaire du numéro

Éditeur(s)

Institut d'histoire de l'Amérique française

ISSN

0035-2357 (imprimé)

1492-1383 (numérique)

Découvrir la revue

Citer cet article

Trudel, M. (1966). Des sujets de recherche, pour un siècle ou deux.... Revue

d'histoire de l'Amérique française, 20(2), 228-235.

https://doi.org/10.7202/302567ar d'utilisation que vous pouvez consulter en ligne.

https://apropos.erudit.org/fr/usagers/politique-dutilisation/ 


\section{DES SUJETS DE RECHERCHE, POUR UN SIĖCLE OU DEUX ...*}

Bien qu'elle soit de pauvres ressources, en regard des colonies anglaises, la Nouvelle-France possède, en 1760 , les cadres qui lui sont nécessaires. Même si elle présente, sous bien des rapports, des traits qui sont communs à toutes les autres sociétés transplantées d'Europe (anglaise, hollandaise, suédoise ou espagnole), la société du Saint-Laurent est une société proprement canadienne; autant que la langue, la religion et le mode de gouvernement, la mentalité, les habitudes de vie, le sentiment d'une commune aventure ont fait des Canadiens un groupe bien déterminé, qui réagit comme tel en face des autres groupes, même en face d'un groupe aussi voisin que celui des Acadiens.

Parce que, préoccupé comme on l'est en Europe de la seule économie européenne, on n'a point travaillé à en faire un corps qui subsiste par lui-même, la Nouvelle-France ne peut survivre sans une métropole. Or voici que le cours de sa vie normale change brusquement: cette société française, devenue américaine et canadienne, mais toujours conditionnée dans son existence par l'Europe, perd sa métropole naturelle et doit s'intégrer dans ce monde anglais contre qui elle luttait depuis trois quarts de siècle. Cette nouvelle métropole, il est vrai, ressemble, à bien des points de vue, à l'ancienne: spirituellement, elle est chrétienne et se dit catholique; l'Église et l'État, le trône et l'autel ont l'un avec l'autre, les mêmes relations étroites; socialement, cette nouvelle métropole est hiérarchique; politiquement, elle est monarchique, et le roi d'Angleterre per-

\footnotetext{
* Conclusion d'un volume, Initiation à l'étude de la Nouvelle-France: histoire et institutions, qui paraîtra au cours de l'automne, chez Holt, Rinehart \& Winston (Montréal). Tous droits réservés.
} 
siste à porter le titre de roi de France; économiquement, Londres est surtout intéressée, comme on l'était à Paris, aux grandes entreprises de la fourrure. Toutefois, à côté de ces similitudes, il y a des différences qui peuvent influer profondément sur l'avenir de la société canadienne: l'Angleterre est séparée de Rome et son roi a juré, lors du sacre, de détruire le papisme; elle est d'une langue et d'une culture autres que celles de la France; sa monarchie repose sur la libre acceptation par le peuple; la société anglaise n'est pas cette société paternaliste qui ordonne tout à l'avance et pourvoit à tout, mais une société qui pratique la libre initiative: la tenure anglaise et le régime seigneurial français illustrent bien les différences extrêmes de ces deux sociétés.

La Nouvelle-France s'est désintégrée après la conquête, mais il reste donc, dans le Saint-Laurent, une société française, pourvue de cadres et qui pratique déjà une forme traditionnelle de vie. Quel sort lui réserve son intégration dans l'empire anglais ? La société canadienne a-t-elle eu conscience de commencer soudain à vivre un drame ? Ce passage d'un monde français à un monde anglais a-t-il eu le caractère tragique qu'on lui prête?

Pour répondre à cette question comme pour mesurer le poids de la conquête sur notre destin, il nous faudrait connaître, de cette société du régime français, bien plus que ses cadres. Or cette société, nous la connaissons encore bien peu; de grands problèmes continuent à se poser, sans que les historiens n'aient encore apporté de réponses satisfaisantes. Les sujets d'étude sont, en ce domaine, d'une grande abondance.

Quelles ont été, par exemple, les relations entre cette société et celle de France? Quelles relations ont eues entre eux les divers groupes du peuplement français de l'Amérique ? Dans quelle mesure leur dispersion et leur isolement ont-ils conditionné leur mentalité ? Et que sait-on des premiers éléments qui sont venus constituer la population originelle ? Si l'on connaît assez bien le nombre que chaque province de France a pu fournir, nous ignorons les conditions particulières qui ont 
poussé les divers groupes de Français à émigrer; comme nous ignorons toujours ce que valaient ces gens de métiers, ce qu'étaient ces recrues militaires qui se transforment en agriculteurs, de quel milieu venaient les "filles du roi" et comment elles se sont adaptées à ces inconnus qu'on leur fait rapidement épouser, quel a été le comportement de ceux qu'on implante ici en guise de punition, quel a été ici le nombre et le rôle des huguenots après la révocation de l'édit de Nantes. Dans ce peuplement du régime français, il reste aussi à apprécier l'apport des Européens non français et celui des Amérindiens: sera-t-il jamais possible de savoir dans quelle proportion les Canadiens français d'aujourd'hui sont de descendance anglaise ou sauvage ? De cette population, enfin, nous connaissons mal les couches dont elle est stratifiée, comment les groupes sociaux se sont formés, puis transformés, quels échanges ils ont eus, ce qu'est la noblesse en Nouvelle-France, ce qu'est la bourgeoisie, ce qu'il faut entendre par peuple, comment on s'élève d'un groupe social et comment on y retombe, ou encore ce qu'est la société canadienne en regard de la société française d'Europe.

Le mécanisme de gouvernement nous est bien connu, mais est-ce tellement certain qu'il a fonctionné comme il était conçu ? Il y a loin, souvent, de la théorie à la pratique, de la loi à l'exécution. Au reste, nous aimerions bien davantage savoir le comportement social des fonctionnaires de l'État: pauvrement rémunérés, de quoi vivaient-ils donc ? dans quelle mesure le service public, et, partant, le bien commun, en a-t-il souffert? quelle a été la pratique du cumul des charges et quels en ont été les inconvénients ? et surtout, nous attendons toujours qu'on étudie le comportement de la masse des gens en face de ce régime d'absolutisme que vient corriger ou alourdir le paternalisme: il nous sera alors plus facile d'observer, sous le régime anglais, l'adaptation de cette masse à la libre initiative et au régime parlementaire.

Nous croyons en savoir assez long sur l'organisation militaire, mais que savons-nous des conditions de vie des officiers et soldats de l'armée régulière, de la façon dont ils ont agi 
dans la société et dont celle-ci les a traités, de l'influence que leur présence a exercée sur la vie économique ? que savons-nous du rôle et des conditions de service des miliciens ? et que savonsnous de ce petit chef paroissial, le capitaine de milice ?

Ce n'est, en somme, que de nos jours, qu'on a abordé le régime seigneurial avec des préoccupations d'histoire sociale. Ici, le champ d'étude est vaste. On attend la carte seigneuriale définitive, qui permettra, en reconstituant sur papier l'ensemble du domaine, d'apprécier les avantages et les inconvénients matériels de ce régime en un milieu donné. Le rôle que les laïcs et les institutions religieuses ont joué dans le développement du système, les droits et les devoirs que seigneurs et censitaires ont réellement exercés, l'importance que ces droits et devoirs ont eue dans la vie sociale et dans la vie économique, l'histoire de la suppression du régime en 1854 et de sa tardive disparition; enfin, dans quelle mesure deux siècles de vie seigneuriale ont $\mathrm{pu}$ façonner un certain type de Canadien français : voilà autant de problèmes, et il y en a d'autres encore, sur lesquels les historiens ont à se pencher.

De l'histoire économique du régime français, la plus grande partie reste encore à faire, malgré certaines études d'envergure. Nous attendons encore les travaux méthodiques sur le système monétaire (à lui seul, le papier-monnaie est un champ immense de recherche), sur l'administration des finances, sur les communications intérieures. On a beaucoup parlé de commerce, surtout du commerce des fourrures, mais que sait-on jusqu'ici de ceux qui l'ont pratiqué ? Il nous faudrait d'abord un inventaire des marchands, puisque, sur eux, nous nous posons tant de questions: origine, point de départ, volume des affaires, train de vie, richesse réelle, rang social, mentalité, visées aristocratiques, influence sur le destin de la colonie, ascension ou décadence, survivance par leurs fils ou extinction; peut-être, lorsqu'on aura répondu à ces questions, pourra-t-on mieux expliquer pourquoi nos marchands perdent la direction des affaires, à la fin du $18 \mathrm{e}$ siècle. Il nous restera encore à observer le mécanisme de tout le petit commerce: marchands 
détaillants, coureurs de côtes, marchés et boutiques, marchandises en circulation, variation des prix.

Sur l'industrie, on n'a toujours qu'un rapide inventaire, plutôt chronologique; il ne répond plus aux questions d'aujourd'hui: pourquoi, par exemple, sous le régime français, ce pays riche en bois n'a pas soutenu une construction navale aussi abondante que celle de la Nouvelle-Angleterre? Des forges Saint-Maurice, notre seule industrie lourde, il faudra faire une histoire économique et sociale plus poussée que celles que nous avons. Que savons-nous des pêcheries du fleuve et du golfe, du volume d'affaires des marchands de Québec en ces entreprises, des hommes qui se livraient à cette pêche ? Pourquoi les petites industries de première nécessité ont-elles si mal réussi ? Que savons-nous de la pratique et du rôle des industries domestiques ? Qui étudiera, enfin, le système des poids et mesures, sans la connaissance duquel on ne peut faire d'étude économique qui soit valable?

L'agriculture est un autre champ immense, ouvert à la recherche, maintenant que parler de l'agriculture ne veut pas dire la chanter. On n'a pas encore raconté l'évolution de ces immigrants qui, tirés des métiers ou des cadres militaires, se sont faits habitants; ni montré de quel rapport a pu être l'agriculture aux $17 \mathrm{e}$ et $18 \mathrm{e}$ siècles, ni décrit l'état réel de l'agriculture au moment où la colonie laurentienne sera contrainte par les nouveaux maîtres, à devenir une colonie agricole. Et nous connaissons fort mal l'habitant moyen: de quoi vivaitil ? que mangeait-il ? quelles étaient sa situation économique, sa mentalité, ses habitudes sociales?

Nous savons assez bien comment, dans l'ensemble, a fonctionné l'administration de la justice, mais les détails du rouage nous échappent toujours. Il reste bien des avenues à ouvrir: nous ne savons rien de la justice ecclésiastique, parce que personne encore n'a eu accès aux dossiers; faute de documents, nous ne sommes guère renseignés sur les diverses cours seigneuriales; on n'a pas encore écrit l'histoire des praticiens ni celle des arpenteurs. Il faudra aussi raconter l'adaptation de 
la Coutume de Paris aux exigences d'un pays neuf et sa transformation en ce qu'on a appelé lois du Canada.

L'histoire de l'hygiène du régime français n'est pas faite, non plus que celle de l'assistance sociale aux pauvres et aux bâtards; sur la médecine et sur la chirurgie, nous avons des études qui ne répondent pas aux questions de l'histoire économique et sociale.

Mentionner l'histoire religieuse, c'est faire surgir un nombre étonnant de problèmes historiques. Quelle est la situation exacte de l'Église par rapport à l'État ? comment se comportent les évêques vis-à-vis les autorités laïques ? De ces évêques, il faut remettre à jour les biographies, étudier leurs origines sociales, leurs relations avec le clergé. Du Chapitre, communauté de contemplatifs, la vie n'a pas été racontée. Du clergé séculier, on connaît peu le régime de vie, les ressources personnelles (qu'ont rapporté, par exemple, les dîmes ?), la valeur intellectuelle, le comportement social, le phénomène de la canadianisation. Le clergé régulier n'a pas été tellement étudié: les Messieurs du Séminaire de Québec n'ont pas encore leur historien; ceux du Séminaire de Montréal attendent que leur histoire soit renouvelée selon les exigences actuelles; le rôle des Prêtres du Saint-Esprit en Acadie n'a été raconté que dans un climat de revendication; on n'a fait l'histoire des Récollets que pour la région des Trois-Rivières; on n'a rien sur les Capucins de la Nouvelle-France. Les Jésuites ont été les plus abondamment servis par l'historiographie, mais tout n'est pas dit, puisque ce que l'on sait le mieux, c'est le commencement, c'est-àdire le $17 \mathrm{e}$ siècle.

Si nous passons aux auxiliaires de ce clergé, constatons de grands vides. Il reste à écrire sur les Frères Charron, hospitaliers et instituteurs; sur les communautés de femmes, cloîtrées ou séculières, dont les histoires pieuses ou simplement chronologiques qu'on en a faites, laissent de côté les problèmes économiques et sociaux.

Sur l'institution paroissiale, on a beaucoup écrit, mais, à part les éloges classiques, peu sur le curé; le rôle considérable 
qu'on lui attribue n'a pas encore été démontré: comment ce chef religieux de la paroisse s'est-il comporté ? Quelles ont été ses relations avec le seigneur, avec le capitaine de milice (chef civil et militaire), avec les paroissiens ? Heureux qui mettra la main sur des prônes et sermons du régime français. Et la Fabrique ? Là aussi, nous souhaitons de connaître la part réelle qu'ont eue les marguilliers dans l'administration, comme nous attendons, en ce domaine, une vaste étude économique. D'ailleurs, nous espérons qu'un jour on essaiera d'évaluer l'importance qu'a eue sur la vie économique de la Nouvelle-France, l'ensemble des institutions qui relèvent de l'Église.

Les questions à étudier ne sont pas moins nombreuses, quand on en arrive à la pratique religieuse. A quelles sources s'est alimentée la vie religieuse; quels effets ont produit, sur sa mentalité, la Contre-Réforme catholique, les querelles jansénistes et piétistes; quelle a été l'influence de la Compagnie du Saint-Sacrement: comment, en somme, peut-on qualifier la spiritualité de la Nouvelle-France ? Les règles de la pratique religieuse ne sont pas mieux connues: des sept sacrements, l'exercice de deux seulement (la Pénitence et le Mariage) a été décrit dans le détail; l'observance des dimanches et fêtes d'obligation, ainsi que l'abstinence et le carême méritent des études fouillées: il faut non seulement les décrire, mais aussi en rappeler les implications socio-économiques.

Enfin, la vie intellectuelle de la Nouvelle-France est un autre domaine dans lequel il y a encore beaucoup à faire. On sait peu de chose de l'éducation dans le monde rural, et rien sur les écoles de Fabrique; du Collège des Jésuites, on n'a pas encore fait l'histoire. Il existe un inventaire général des bibliothèques, mais il reste à le compléter et à faire une analyse méthodique du contenu. Nous n'avons de la littérature écrite aucune étude générale satisfaisante; quant à la littérature orale, sur laquelle on accumule beaucoup de documentation, nous souhaitons qu'on en publie, un jour, une interprétation qui aide à la connaissance du régime français. Sur les arts, les monographies se font de plus en plus nombreuses; dans les 
sciences, on a commencé à aborder les relations de nos scientifiques avec ceux d'Europe, mais, en ces deux domaines, font aussi défaut les synthèses.

Voilà bien des questions que le présent continue de poser au passé ! Les nouvelles recrues d'historiens apporteront, peu à peu, des réponses; puis, comme le veut la loi du progrès, lorsque ces réponses auront été données, peut-être faudra-t-il alors, pour se conformer aux exigences d'une nouvelle époque, tout recommencer...

MARCEL TRUdeL, professeur à l'Université d'Ottawa

Un volume à retenir :

Nous recommandons, de M. Fernand Ouellet, Histoire économique et sociale, 1760-1850: structures et conjoncture. Préface de Robert Mandrou, directeur à l'Ecole pratique des Hautes Etudes, Paris. Editions Fides, 245 est, Dorchester, Montréal 18, Qué.

L'ouvrage de M. Ouellet a été publié sous la direction du Centre de Recherches en Histoire économique du Canada français. L'Ecole des Hautes Etudes commerciales de Montréal et l'Université Sir George Williams ont mis sur pied ce Centre de recherches en histoire économique. Tout en se consacrant spécialement à l'histoire économique du Canada français, ce Centre de recherches ne néglige pas l'histoire des Amériques et ses entrecroisements d'influences.

LIONEL GROULX, ptre 